\title{
The TileCal/ATLAS calorimeter calibration systems
}

\author{
J. Carvalho \\ LIP-Coimbra, University of Coimbra, 3004-516 Coimbra, Portugal \\ E-mail: jcarlos@coimbra.lip.pt \\ (on behalf of the ATLAS Collaboration)
}

\begin{abstract}
The calibration and monitoring systems of the ATLAS hadronic tile calorimeter (TileCal), are presented. Special attention is given to the experience gained so far with the results obtained on the analysis of the LHC collision data.

Keywords: hadron calorimetry; calibration; monitoring.
\end{abstract}

\section{Introduction}

The ATLAS central hadronic calorimeter ${ }^{1}$ is a sampling calorimeter built from steel absorber plates and scintillator tiles. It consists of three cylinders along its axis, each divided in 64 wedges in $\phi$, and it covers a pseudorapidity up to $|\eta|=1.7$. Each tile is read out by two wavelength shifting fibers, coupled to photomultiplier tubes (PMT). The fibers are grouped in bundles forming projective towers pointing to the interaction region. The calorimeter is readout by $10 \mathrm{k}$ channels for $5 \mathrm{k}$ cells, of size $\Delta \eta \times \Delta \phi=0.1 \times 0.1$ with twice coarser granularity in $\eta$ for the outer of the three longitudinal layers.

Different systems are used to calibrate and monitor the calorimeter. The charge injection system is used to calibrate the relative response of the PMT signal readout electronics and to track any variation with time. The laser system is designed to calibrate and monitor the response of the PMTs, in particular the stability of their gains, their global linearity and for timing studies. A Cesium radioactive source system is designed to measure the quality of the optical response of each calorimeter cell, to equalize the signal response and to monitor it with time. Finally the current produced by minimum bias interactions, integrated over thousands of bunch crossings, is used to continuously monitor the calorimeter response during collision runs. This system is called the Minimum Bias (MB) calibration system. About $12 \%$ of the modules were previously calibrated in a testbeam with 
electrons, muons and pions. ${ }^{2}$

\section{Calibration and monitoring of the TileCal}

Each TileCal cell can be divided, for monitoring and calibration purposes, into: the optical part (the scintillating tiles and the wavelength shifting fibers); the PMT that converts and amplifies the optical signal; and the readout electronics that shape, amplify and digitize the signal. For each one of these parts a dedicated monitoring and calibration system was designed and built as described in the next sections. Fig.1 shows a diagram of the sequence of the TileCal optical and electronic readout, and the inputs of the calibration and monitoring systems.

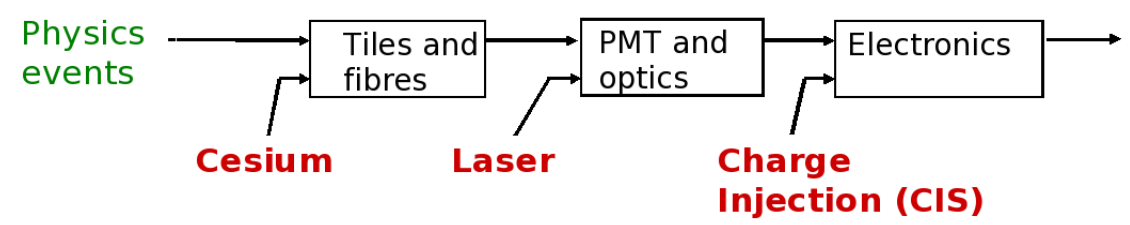

Fig. 1. Diagram of the optical and electronic readout, and of the calibration and monitoring systems.

\subsection{Charge Injection System}

The charge injection system (CIS) is designed to calibrate the relative response of the pulse readout electronics for all calorimeter PMTs and to monitor their variations with time. Each PMT channel has two analogue paths (bi-gain), the high and low gain ( 82 counts $/ \mathrm{pC}$ and 1.3 counts $/ \mathrm{pC}$, respectively), digitized by a 10-bit $\mathrm{ADC}$, covering a range of $800 \mathrm{pC}$ (corresponding to a energy deposition of about $700 \mathrm{GeV}$ ).

Each channel is equipped with calibration capacitors, charged from a high precision voltage source and discharged into the input electronics. The measurement of the charge injected at the input stage of the electronic channels allows to obtain the CIS constants used to convert the signal from ADC counts to pC. ${ }^{3}$

In ATLAS there are periodic CIS runs scanning the full dynamic range during beam-off periods (between LHC fills and maintenance periods).

The variation of the calibration constants for individual ADC channels over the first half of 2011 data taking period has a RMS of about $0.1 \%$ and 
channels with a variation greater than $1 \%$ are tagged for recalibration.

\subsection{Laser system}

The laser system was designed to calibrate and monitor the PMT response. It is also used to check the PMT linearity, for studies on pulse saturation recovery and in calorimeter timing.

A laser is used to produce pulses with a wavelength of $532 \mathrm{~nm}$ and a 10 ns width, synchronized with the bunch-crossing clock. The light is split, with part of it being sent to a set of precise photodiodes for relative intensity monitoring, and the remaining being delivered to each calorimeter PMT.

The short and long term monitoring of the PMT gain stability is done in special calibration runs from the ratio of the charge measured by each PMT to the photodiodes response. ${ }^{4}$

The linearity of the PMT can be studied varying, in a well defined range, the intensity of the light delivered.

Fig. 2 shows the result of a PMT high voltage (HV) scan to check the system sensitivity to the gain change, since the PMT response in this case scales linearly to the applied HV for small HV changes. The agreement between the measured and the expected gain variation is very good, showing that the gain monitoring sensitivity is adequate for the response follow-up in time.

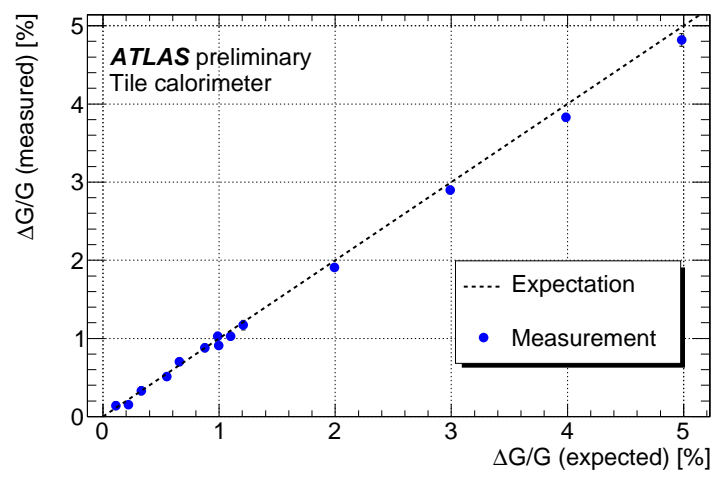

Fig. 2. Measured relative gain variation as a function of the expected relative gain variation, from a PMT high voltage (HV) scan. 


\subsection{Cesium source system}

Cs calibration is designed to determine the quality of the optical response of each calorimeter cell, to adjust the PMT high voltage in order to equalize the response from all cells and to monitor it with time. ${ }^{5}$ The system uses a ${ }^{137} \mathrm{Cs} \gamma$ source which moves perpendicularly to the tiles' surface through a hole in the scintillating tiles. This source is transported by a hydraulic system in a series of straight paths along the calorimeter modules, so it passes through holes in every single scintillating tile and absorber plate. The current from each PMT is measured with an integrator during the time of the passage of the source capsule through each cell. ${ }^{6,7}$

In ATLAS the Cesium system is used to monitor the long term stability of the calorimeter. It allows not only to monitor the PMT stability but also to detect bad tile-fiber couplings, scintillator aging and optical problems in general.

Fig. 3 shows the TileCal response to the Cesium source as a function of time, in all four partitions (the activity of the three sources used are different). The response follows, in a reasonable way, the expected Cesium activity decay curve in the two years shown. The small deviation is due to the expected up-drift of the PMT gain during the initial phase of light exposure.

\subsection{Minimum bias events}

The minimum bias (MB) events in ATLAS are inelastic pp collisions with low momentum transfer, whose rate is proportional to the LHC luminosity. ${ }^{7}$ These events produce a non-negligible occupancy of the TileCal cells, with rates uniform in the azimuthal angle $\phi$ and moderately dependent on the pseudo-rapidity $\eta$. Since the MB current, averaged over milliseconds, is almost constant and proportional to the interaction rate, it is used to monitor the calorimeter response and the relative luminosity during physics runs.

Fig. 4 shows the average anode current for a TileCal cell as a function of the instantaneous luminosity, in 2010. The correlation is excellent, being linear within $0.5 \%$ for higher luminosity. The modulation on the deviation from linearity is due to systematic errors not yet taken into account on the measurement.

\section{Conclusions}

The ATLAS hadronic calorimeter monitoring and calibration systems were presented. The CIS, laser and Cesium allow to calibrate and to monitor the 


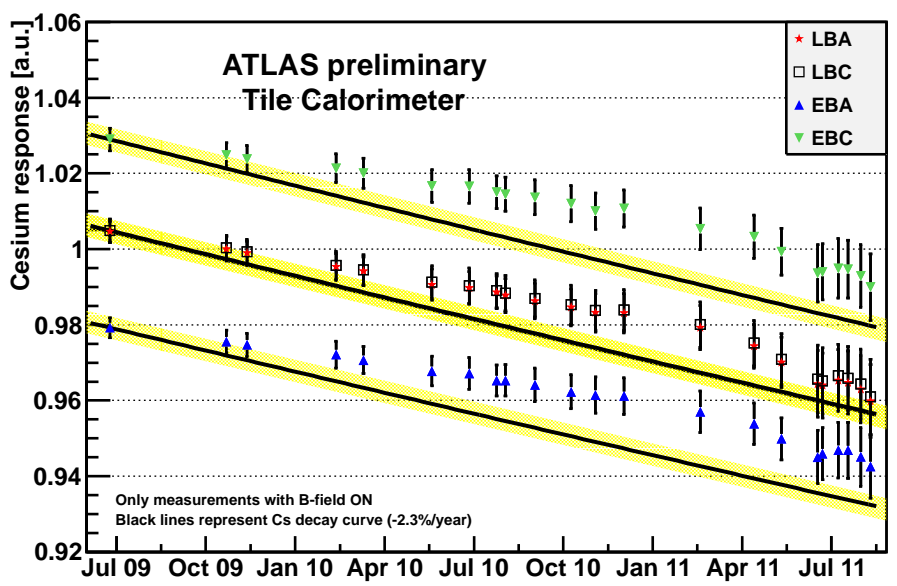

Fig. 3. TileCal response to the Cesium source as a function of time, in all the four partitions. The black lines represent the expected values considering the Cs decay lifetime (decrease of $2.3 \%$ per year).

calorimeter response with a $0.5-1 \%$ precision.

The different calibration and monitoring systems have been commissioned, and perform according to the design requirements while being exploited during data-taking and regular calibration runs. These systems are fundamental to reach the design parameters on the calorimetry energy resolution and energy scale linearity and stability.

\section{References}

1. ATLAS/Tile Calorimeter Collaboration "Tile Calorimeter - Technical Design Report" CERN/LHCC 96-42, 1996.

The ATLAS Collaboration, G. Aad et al., "Readiness of the ATLAS Tile Calorimeter for LHC collisions" Eur. Phys. J. C71 (2011) 1193-1236.

2. P. Adragna et al. "Testbeam studies of production modules of the ATLAS Tile Calorimeter", Nucl. Instr. Meth. A606 (2009) 362-394.

3. K.J. Anderson et al. "Design of the Front-end Analog Electronics for the ATLAS Tile Calorimeter" Nucl. Instr. Meth. A551 (2005) 469-476.

4. V. Giangiobbe "The TileCal Laser calibration system" ATL-TILECALPROC-2011-007, CERN, 2011.

5. E. Starchenko et al. "Cesium monitoring system for ATLAS Tile Hadron Calorimeter" Nucl. Instr. Meth. A494 (2002) 381.

6. N. Shalanda et al. "Radioactive source control and electronics for the AT- 

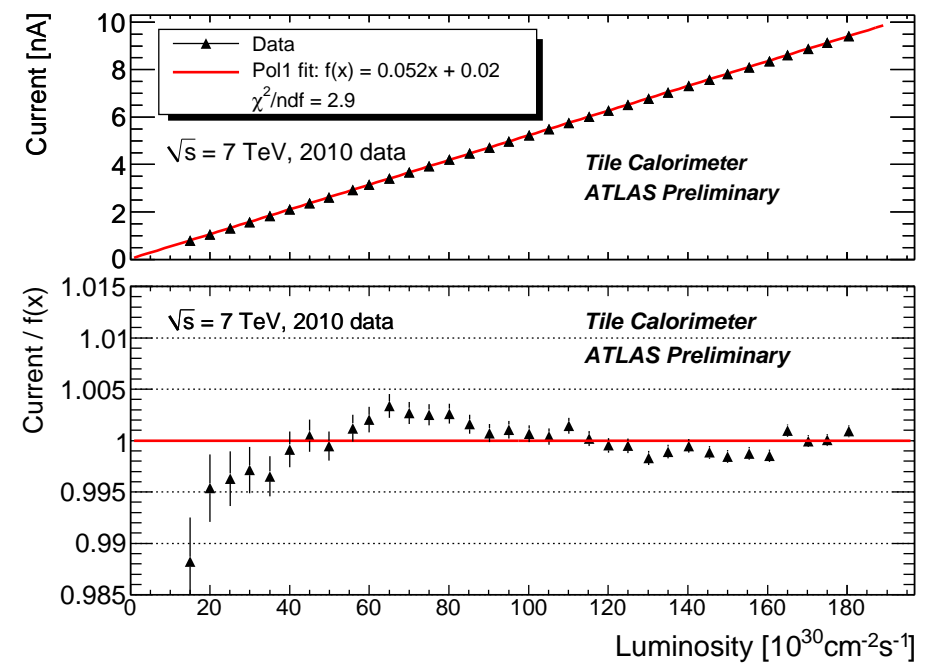

Fig. 4. Average anode current for a TileCal cell as a function of the instant luminosity, in 2011. The bottom plot shows the deviation from the linear behaviour.

LAS Tile Calorimeter cesium calibration system", Nucl. Instr. an Meth. A508 (2003) 276.

7. G. Gonzalez Parra "Integrator based readout in Tile Calorimeter of the ATLAS experiment" ATL-TILECAL-PROC-2011-010, CERN, 2011. 